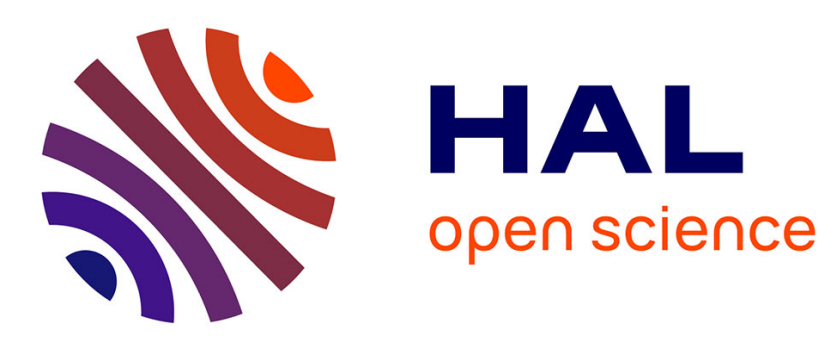

\title{
Le sémantisme propre des cadres prédicatifs et la polysémie des verbes de production de parole
}

\author{
Morgane Sénéchal, Jacques François
}

\section{To cite this version:}

Morgane Sénéchal, Jacques François. Le sémantisme propre des cadres prédicatifs et la polysémie des verbes de production de parole. Colloque: La prédication, Nov 2004, Aix-en-Provence, France. pp.325-335, 10.1163/19589514-31-32-01-900000030 . hal-00012477

\section{HAL Id: hal-00012477 \\ https://hal.science/hal-00012477}

Submitted on 24 Oct 2005

HAL is a multi-disciplinary open access archive for the deposit and dissemination of scientific research documents, whether they are published or not. The documents may come from teaching and research institutions in France or abroad, or from public or private research centers.
L'archive ouverte pluridisciplinaire HAL, est destinée au dépôt et à la diffusion de documents scientifiques de niveau recherche, publiés ou non, émanant des établissements d'enseignement et de recherche français ou étrangers, des laboratoires publics ou privés. 


\title{
LE SEMANTISME PROPRE DES CADRES PREDICATIFS ET LA POLYSEMIE DES VERBES DE PRODUCTION DE PAROLE
}

\author{
Jacques FRANÇOIS ${ }^{1}$ \\ Morgane SENECHAL ${ }^{2}$
}

\section{RESUME $^{3}$}

Dans cet article, nous nous proposons de tester sur une classe de verbes français la thèse d'Adele Goldberg (1995) selon laquelle certains types de structures argumentales (appelées ici «cadres prédicatifs » dans le prolongement de François 2003) présentent un sémantisme propre, en sorte que différents types de polysémie verbale - que D. Willems (2005) classe comme "réguliers" et « syntaxiques» - sont analysables en termes de greffe entre deux cadres prédicatifs, l'un primairement attaché au verbe et l'autre fonctionnant comme greffon. Nous nous attachons particulièrement à la greffe d'une construction verbale à un cadre prédicatif divalent (lui-même éventuellement issu de la greffe d'un actant à un cadre prédicatif monovalent) dans le domaine des verbes de production de parole, par exemple : Marie crie (qch) (à qn) > Marie crie à qn de faire qch; Paul demande (qch) (à qn) > Paul demande à Marie de faire qch > Cela demande qch \{un effort / de la bonne volonté / etc.\} à Marie.

I. LA NOTION DE 'CONSTRUCTION' APPLIQUEE AUX STRUCTURES ARGUMENTALES PAR ADELE GOLDBERG

Les « grammaires de construction » sont issues au début des années 90 des travaux fondateurs de Ch. Fillmore (cf. Fillmore, Kay \& Connors 1988), R. Langacker (cf. 1987, 1991) et G. Lakoff (cf. 1987). Deux variantes semblent actuellement se distinguer: l'une, plus orientée vers les traitements automati-

\footnotetext{
1 CRISCO, FRE 2805 Université de Caen, jacques.francois@crisco.unicaen.fr

2 CRISCO, FRE 2805 Université de Caen, morg.sen@wanadoo.fr

${ }^{3}$ Une version de cet article détaillée et largement illustrée d'extraits du corpus est disponible sur le site du CRISCO : www.crisco.unicaen.fr/cahiers/ sous la forme du Cahier du CRISCO 19.
} 
ques, se rapproche formellement de la Head Driven Phrase Structure Grammar (HPSG), cf. Kay (1997), l'autre plus directement inspirée par Langacker et Lakoff, trouve une expression vigoureuse dans les travaux récents de W. Croft (1998, 2001, Croft \& Cruse 2004 : chapitre 10). Selon Croft (1998 : 90-91, notre traduction) :

« Jusqu'à un certain point, tout mot peut être employé en principe dans n'importe quelle construction. De ce fait les patrons distributionnels n'établissent pas de catégories grammaticales au sens strict. Ce qui compte c'est l'interprétation sémantique d'un mot dans une construction particulière (..) cette interaction entre les constructions grammaticales et les mots que les locuteurs y insèrent est la source de la richesse et de la flexibilité du langage comme moyen de communiquer le vécu. La flexibilité de la grammaire est limitée à un certain point par nos attentes sur ce à quoi ressemble le monde et sur la manière dont différents types d'événements peuvent être construits mentalement de manière plausible ».

A. Goldberg $(1995,1998,1999,2002)$ applique cette vision 'flexible' ou 'élastique' de la grammaire aux structures argumentales. Elle montre en particulier comment le verbe anglais sneeze (éternuer), fondamentalement intransitif et destiné à exprimer une classe d'événements incontrôlés, peut accepter la greffe d'un cadre prédicatif de causation de mouvement, cf. (1).

(1) Pat sneezed the foam off the cappuccino.

Des deux valeurs participatives fondamentalement véhiculées par sneezel [N1+hum ], la valeur [-contrôlé] subsiste, mais la valeur [-causatif] est inversée, l'éternuement de Pat entraînant l'éjection de la mousse du cappuccino. La greffe peut se visualiser sous la forme (2).

(2)

\begin{tabular}{|c|c|c|c|}
\hline $\begin{array}{l}\text { Cadre prédicatif source } \\
\text { Cadre prédicatif greffon }\end{array}$ & $\begin{array}{l}\text { N1:qn } \\
\text { N1 }\end{array}$ & $\begin{array}{l}\text { sneeze- } \\
\mathrm{V}+\mathrm{mvt}\end{array}$ & Oblique: off $\mathrm{N} 3$ \\
\hline $\begin{array}{l}\text { Produit de la greffe } \\
\text { Illustration }\end{array}$ & $\begin{array}{l}\text { N1:qn } \\
\text { Pat }\end{array}$ & $\begin{array}{ll}\text { sneeze- } & \mathrm{N} 2+\text { conc } \\
\text { sneezed } & \text { the foam }\end{array}$ & $\begin{array}{l}\text { Oblique: off } \mathrm{N} 3 \\
\text { off the cappucino }\end{array}$ \\
\hline
\end{tabular}

L'application au français de la notion de «construction » dans le domaine argumental telle qu'elle est exploitée par Goldberg (1995), implique à notre avis trois hypothèses :

i. à chaque verbe "polysémique-syntaxique $»^{5}$ on peut associer un type primaire de prédication (type rencontré le plus fréquemment par les locuteurs natifs et donc le plus disponible cognitivement);

${ }^{4}$ cf. Levin B. \& Rappaport Hovav M.(1995) « The elasticity of verb meaning », Proceeding of the Tenth Annual Conference of the Israel Association for Theoretical Linguistics (IATL2), cité par Willems (2005).

Sous définirons un verbe «polysémique-syntaxique» comme un verbe présentant un éventail de sens (sa polysémie) et un éventail de cadres prédicatifs (sa "polytaxie») corrélés de sorte que chaque cadre prédicatif soit associable à un sens particulier (sans que l'inverse soit nécessairement vrai car par ex. une action peut être exprimée par une 
ii. certains types de cadre prédicatif sont dotés d'un sémantisme autonome (que nous désignerons comme des « constructions prédicatives »);

iii. certaines prédication primaire se laissent greffer une (ou plusieurs) construction(s) prédicative(s).

C'est ce que cherchent à illustrer respectivement les figures 1 et 2 à partir des phrases (3) et (4).

(3) La mère dit aux enfants de se tenir tranquilles.

(4) La résolution du problème demande beaucoup de temps (aux élèves).

Le prédicat verbal dire est considéré comme primairement associé à un cadre prédicatif du type [N1: qn $<\mathrm{x}>\mathrm{V}$ N1: qch $<$ production verbale : $>$ $>$ (à N2: $\mathrm{qn}<\mathrm{y}>$ )] qui distribue trois rôles : un locuteur $<\mathrm{x}>$, un allocutaire $<\mathrm{z}>$ et un contenu $<y>$, lesquels spécifient conjointement un acte de parole déclaratif. La greffe d'un cadre prédicatif habituellement associé à des verbes d'ordre ou requête tels que ordonner, recommander, conseiller ou suggérer $[\mathrm{N} 1$ : qn $<\mathrm{x}>\ldots \mathrm{V}$ $\grave{a} \mathrm{~N} 1: \mathrm{qn}<\mathrm{y}>$ de INF $:$ action $<\mathrm{a}>$ ] convertit une prédication source d'acte de $\overline{\mathrm{pa}}-$ role déclaratif en une prédication résultante d'acte de parole instructif ( ordre ou requête).

Figure 1 : Genèse par greffe d'une prédication d'acte de parole instructif à l'aide du prédicat verbal dire

\begin{tabular}{|c|c|c|c|c|}
\hline $\begin{array}{l}\text { prédication } \\
\text { source }\end{array}$ & $\begin{array}{c}\mathrm{N}: \\
\mathrm{qn}<\mathrm{x}> \\
\text { La mère }\end{array}$ & dit & $\begin{array}{c}\mathrm{N} 1: \text { qch } \\
\text { <prod.verbale : }> \\
\text { quelques mots }\end{array}$ & $\begin{array}{l}(\mathrm{à} \mathrm{N} 2: \mathrm{qn}<\mathrm{y}>) \\
(\text { aux enfants) }\end{array}$ \\
\hline $\begin{array}{l}\text { cadre pré- } \\
\text { dicatif } \\
\text { greffon }\end{array}$ & $\begin{array}{c}\text { N1: } \\
\text { qn<y> }\end{array}$ & $\begin{array}{c}\downarrow \\
\text { \{ordonne, } \\
\text { recommande, } \\
\text { conseille, } \\
\text { suggère\} }\end{array}$ & $\grave{a} \mathrm{~N}: \mathrm{qn}<\mathrm{y}>$ & de IN : action $<\mathrm{a}>$ \\
\hline $\begin{array}{l}\text { prédication } \\
\text { résultante }\end{array}$ & $\begin{array}{c}\mathrm{N} 1: \\
\mathrm{qn}<\mathrm{x}> \\
\text { La mère }\end{array}$ & Dogation & $\begin{array}{l}\dot{a} \mathrm{~N} 2: \mathrm{qn}<\mathrm{y}> \\
\text { aux enfants }\end{array}$ & $\begin{array}{c}\text { de IN : action }<\mathrm{a}> \\
\text { de se tenir tran- } \\
\text { quilles }\end{array}$ \\
\hline
\end{tabular}

Dans ce premier cas (3), la greffe ne change pas le domaine conceptuel de la prédication verbale, celui de la production de parole. Dans le second (4), la greffe sur la prédication primaire [N1: qn $<\mathrm{x}>$ demande $_{\mathrm{V}} \mathrm{N}: \mathrm{qch}<\mathrm{y}>\grave{a} \quad \mathrm{~N} 3$ : $\mathrm{qn}<\mathrm{z}>$ ] d'un cadre prédicatif dont le sujet réfère à une action [N1:

expression prédicative, infinitive ou nominale); cf. Sénéchal (2003) sur la polysémie syntaxique des verbes assister, participer et user.

'Dans la terminologie adoptée ici de la Functional Grammar et de François (2003: 1), une prédication verbale est constituée d'un prédicat verbal et d'un cadre prédicatif. 
$\mathrm{qch}<$ action $: \mathrm{a}>\ldots \mathrm{v} \mathrm{N2:} \mathrm{qch}<\mathrm{y}>(\grave{a} \mathrm{~N} 3: \mathrm{qn}<\mathrm{z}>)]$ a un effet plus drastique, le domaine conceptuel passant de la production de parole à l'expression d'une relation action-moyen, cf. figure 2 .

Figure 2: Genèse par greffe d'une prédication de relation action-moyen à l'aide du prédicat verbal demander

\begin{tabular}{|c|c|c|c|c|}
\hline $\begin{array}{l}\text { prédication } \\
\text { source }\end{array}$ & $\begin{array}{c}\mathrm{N} 1: \mathrm{qn}<\mathrm{x}> \\
\text { Marie }\end{array}$ & demande & $\begin{array}{c}\mathrm{N} 2: \text { qch }<\mathrm{y}> \\
\text { de la patience }\end{array}$ & $\begin{array}{l}\text { à } \mathrm{N} 3: \mathrm{qn}<\mathrm{z}> \\
\text { aux élèves }\end{array}$ \\
\hline $\begin{array}{l}\text { cadre prédi- } \\
\text { catif greffon }\end{array}$ & a : action & $\begin{array}{c}\mathrm{V} \\
\text { \{nécessite, } \\
\text { implique\} }\end{array}$ & qch & $\begin{array}{l}\text { (de la part } \\
\text { de N) }\end{array}$ \\
\hline $\begin{array}{l}\text { prédication } \\
\text { résultante }\end{array}$ & $\begin{array}{l}\mathrm{N} 1: \mathrm{qch}<\mathrm{act}: \mathrm{a}> \\
\text { La résolution } \\
\text { du problème }\end{array}$ & demande & $\begin{array}{l}\mathrm{N} 2: \text { qch }<\mathrm{y}> \\
\text { de la patience }\end{array}$ & $\begin{array}{c}(\mathrm{à} \mathrm{N} 3: \text { qn }<\mathrm{z}> \\
\text { aux élèves }\end{array}$ \\
\hline
\end{tabular}

Ce type de polysémie syntaxique touche également plusieurs synonymes de demander: exiger, requérir, réclamer (La résolution du problème demande / exige / requiert / réclame du temps), il présente donc un caractère de " régularité ») selon Apresjan (1974) et Willems (2002, 2005).

\section{LES VERBES DE PRODUCTION DE PAROLE, PRIMAIRES OU DERIVES ?}

Existe-t-il clairement une classe des verbes de «production de parole »? D. Willems $(2005, \S 2.2)$ présente trois verbes français susceptibles d'entrer dans cette classe, mais à la suite d'une greffe syntaxique qui contribue à un chaînage polysémique : glisser est fondamentalement un verbe de mouvement, mais on peut glisser «à son voisin qu'il est temps de partir / qu'il parte / de partir »; rapporter et accorder sont des verbes de donation, mais on peut rapporter à quelqu'un qu'un événement s'est produit, et accorder à son adversaire qu'il a marqué un point [N1 V N2 à N3 $\rightarrow \mathrm{N} 1 \mathrm{~V}$ à $\mathrm{N} 2$ que $\mathbf{P}$ ]. Il est donc clair qu'un verbe peut dénoter une production de parole à titre primaire ou à la suite d'une greffe déclenchant une dérivation de sens. Inversement, en passant de (5) à (5a) ou (5b), nous quittons le domaine de la production de parole, de même qu'entre (6) et (6a).

(5) Marie ne me dit rien.

[(absence d') activité]

(5a) Le silence de Marie ne me dit rien (qui vaille).

[état expérientiel affectif]

\footnotetext{
${ }^{7}$ Il est à noter que dans la prédication résultante de relation action-moyen, demander conserve de son cadre prédicatif source la possibilité d'introduire un complément prépositionnel introduit par $\grave{a}$, alors que les verbes typiques de relation action-moyen, nécessiter et impliquer ne sont compatibles qu'avec un circonstant du type de la part de $\mathrm{N}$ : qn.
} 
(5b) Le nom de Marie me dit quelque chose.

[état expérientiel cognitif]

(6) Je vous demande toute votre attention.

[activité de communication]

(6a) Ce travail demande toute votre attention. [relation action-moyen]

Un troisième type de verbe de production de parole peut dénoter selon le cadre prédicatif des types d'acte de parole différents, comme nous venons de la voir pour dire (Figure 2). Dans ce cas, l'orientation de la chaîne de polysémie (Willems 2005) n'est pas toujours évidente. Si, pour le verbe appeler, (7a) est clairement dérivable sémantiquement de (7) par GREFFE de la construction infinitive, en revanche pour demander on peut hésiter entre la dérivation de $(8 \mathrm{a}:$ requête) à partir de (8 :questionnement) ou la dérivation inverse, car il y a ici SUBSTITUTION entre deux types de complémentation, interrogative indirecte en (8) vs. infinitive en (8a).

(7) L'institutrice appelle les élèves.

[activité de communication : appel]

(7a) L'institutrice appelle les élèves à se lever et à se présenter.

[activité de communication : appel \& requête]

(8) L'institutrice demande aux élèves s'ils ont leur cahier de textes. [activité de communication : questionnement]

(8a) L'institutrice demande aux élèves d'ouvrir leur cahier de textes. [activité de communication : requête]

\section{LES TYPES D'EMPLOI FONDAMENTAUX DES VERBES DE PRODUCTION DE} PAROLE PRIMAIRES

On peut classer les types majeurs d'emploi des verbes de production de parole sur la base de leur cadre prédicatif primaire ${ }^{8}$ :

- les emplois d'élocution de la forme [N1:qn $<x>V]$, où $<x>$ produit des sons (éventuellement signes linguistiques) d'une certaine manière, ex. qn chante, bégaie, parle, zézaie;

- les emplois de déclaration de la forme $[\mathrm{N} 1$ :qn $<\mathrm{x}>\mathrm{V}$ (N2 :qch / que P / Pq / $\mathrm{INF}<\mathrm{e}>$ )] où $<\mathrm{x}>$ fournit à $<\mathrm{y}>$ une information sur un événement ou une situation $<\mathrm{e}>$, ex. qn dit, raconte, explique qch à qn;

- les emplois de requête de la forme $[\mathrm{N} 1: \mathrm{qn}<\mathrm{x}>\mathrm{V}$ N2 $:$ act/chose / à/de INF / que $\operatorname{Psubj}<a>(a ̀ ~ N 3: q n<y>)]$ où $<x>$ fait connaître à son interlocuteur $<y>$ son souhait que $<\mathrm{x}>$ ou $<\mathrm{y}>$ accomplisse une action $<\mathrm{a}>$;

- les emplois d'appel de la forme [N1:qn $<\mathrm{x}>\mathrm{V}$ N2 :qn $<\mathrm{y}>]$ où $<\mathrm{x}>$ entre en communication verbale avec $<\mathrm{y}>$, ex. qn appelle, interpelle, questionne, interroge qn;

\footnotetext{
${ }^{8}$ Abréviations : que $\mathrm{P}$ / Psubj : complétive à l'indicatif / au subjonctif ; Pq : interrogative indirecte. Ce classement s'inspire librement de la typologie des verbes véhiculant un type d'acte de discours proposée par Vanderveken (1988).
} 
- les emplois de questionnement de la forme [N1 :qn $<\mathrm{x}>\mathrm{V}$ à $\mathrm{N} 2: \mathrm{qn}<\mathrm{y}>$ $\mathrm{N} 3$ : qch $/ \mathrm{Pq}<\mathrm{e}>$ ], où $<\mathrm{x}>$ entre en communication verbale avec $<\mathrm{y}>$ pour obtenir de celui-ci une information sur un événement ou une situation $<\mathrm{e}>$, ex. $q n$ demande à qn la date d'un événement / si $\mathrm{P}$;

- les emplois d'appel-déclaration de la forme [N1 :qn $<x>V$ N2 :qn $<y>$ de $\mathrm{N}$ :prop-évén-act /que $\mathrm{P} / \mathrm{Pq}]$ où $<\mathrm{x}>$ interpelle $<\mathrm{y}>$ pour transmettre à celui-ci une information sur un événement ou une situation $<\mathrm{e}>$, ex. qn informe, avertit, prévient, avise qn que $\mathrm{P}$;

- les emplois d'appel-requête de la forme [N1 :qn $<x>$ V N2 :qn $<y>$ à/de INF / que $\operatorname{Psubj}<\mathrm{a}>$ ] où $<\mathrm{x}>$ interpelle $<\mathrm{y}>$ pour faire connaître à celui-ci son souhait que $<\mathrm{x}>$ ou $<\mathrm{y}>$ accomplisse une action $<\mathrm{a}>$, ex. qn invite, pousse, incite, engage qn à INF ; qn prie, implore, supplie, somme qn de INF

- les emplois d'interlocution de la forme [N1 :qn $<\mathrm{x}>\mathrm{V}$ avec $\mathrm{N} 2: \mathrm{qn}<\mathrm{y}>$ ) alternant avec [N1 :qn $<\mathrm{x}>\&$ qn $<\mathrm{y}>\mathrm{V}]$, où $<\mathrm{x}>$ et $<\mathrm{y}>$ entrent en communication verbale sans but spécifié, ex. qn s'entretient, bavarde, parle avec $q n ; q n<\mathrm{x}>$ et $q n<\mathrm{y}>\mathrm{s}$ 'entretiennent, bavardent, parlent (ensemble)

- et enfin les emplois de nomination de la forme [N1 :qn $<\mathrm{x}>\mathrm{VN} 2$ :qch/qn $<\mathrm{y}>$ (comme/de) « $\mathrm{N} »$, où $\langle\mathrm{x}\rangle$ attribue la désignation $\langle\mathrm{N} »$ à $\langle\mathrm{y}\rangle$, ex. qn appelle, nomme qn « $\mathrm{N} »$, désigne qn comme « $\mathrm{N} »$, qualifie qn de « $\mathrm{N} »$.

Une enquête quantitative menée sur cinq verbes de production de parole dans le corpus du journal Le Monde (année 1993) révèle les profils suivants :

- Hurler (102 occurrences retenues) est associé primairement à la production de sons non linguistiques $(58 \%$ dont $11 \%$ hors du champ de la parole, ex. les pneus hurlent) ou indéterminés $(16 \%)$ et secondairement à des emplois de déclaration $(27 \%)$; les emplois relativement ou complétement figés (ex. hurler à la mort) sont très fréquents.

- Curieusement, crier (200 occurrences retenues) a un profil sensiblement différent : les emplois dominants sont des déclarations par citation $(32 \%)$ ou par intégration (31\%), s'y ajoutent des emplois secondaires de production de sons non linguistiques $(17 \%)$ et de déclaration figée (16\%, ex. crier gare, victoire, au loup).

- Pour demander (200 occurrences retenues), dans ce corpus ce sont les emplois de requête qui dominent $(70 \%)$, les emplois de questionnement étant secondaires $(14 \%)$ et les emplois extralinguistiques marginaux (5\%).

- Dire (188 occurrences retenues) présente 98\% d'emplois de déclaration, 2\% d'emplois de questionnement (ex. dire à qn si $\mathrm{P}$ ) et curieusement aucun emploi de requête (dire à qn de INF).

- enfin appeler (200 occurrences retenues) a majoritairement des emplois de nomination (appeler qn « $\mathrm{N} », 51 \%$ ), immédiatement suivis d'emplois d'appelrequête (appeler qn à INF, 42\%).

Pour hurler, crier et appeler les emplois di- et trivalents dérivent de l'emploi monovalent par greffe de cadres prédicatifs habituellement associés à des emplois de déclaration ou de nomination, mais quantitativement, seul hurler a dans ce corpus des emplois monovalents dominants, pour crier ce sont les emplois déclaratifs et pour appeler les emplois dénominatifs qui l'emportent. Pour de- 
mander, deux et pour dire trois types de greffes sont en compétition à partir d'un type d'emploi déjà trivalent ( $q n$ dit/demande qch à qn). Dans ce corpus, l'emploi de requête l'emporte sur l'emploi de questionnement pour demander, tandis que pour dire les emplois déclaratifs écrasent les emplois de questionnement et de requête.

IV.TROIS CADRES PREDICATIFS DES VERBES DE PRODUCTION DE PAROLE SUSCEPTIBLES DE CONSTITUER DES « CONSTRUCTIONS PREDICATIVES »

Notre hypothèse est que les emplois résultant de la greffe d'un cadre prédicatif sur une prédication verbale primaire intègrent le sémantisme propre de ce cadre, mais il reste à illustrer cette hypothèse à partir de cadres prédicatifs effectivement dotés d'un tel sémantisme. Dans ce but, nous avons sélectionné trois cadres (relevant respectivement de la modalité d'appel-requête et de requête, cf. §III) susceptibles de constituer des " CONSTRUCTIONS PREDICATIVES » (pour lesquels on suppose qu'il est possible d'identifier une tendance sémantique) :

Type 1a $\mathrm{N} 1<\mathrm{X}>\mathrm{V} \mathrm{N} 2<\mathrm{Y}>\grave{a} \mathrm{INF}<\mathrm{A}>$

(APPEL-REQUETE)

(9) ... ma grand-mère obligeait sa bonne à enlever la poussière des rainures du plancher avec une épingle à cheveux (M. TOURNIER, 1978, Le coq de bruyère).

Type $1 \mathrm{~b}>\mathrm{N} 1<\mathrm{X}>\mathrm{V} \mathrm{N} 2<\mathrm{Y}>$ de $\mathrm{INF}<\mathrm{A}>$

(APPEL-REQUETE)

(10) Dans le car, je priai Valentin de faire arrêter les pin-pon furieux de la sirène (G. MORDILLAT, 1981, Vive la sociale).

(11) ... ainsi elle empêcha François de rebrousser chemin (R. RADIGUET, 1923, Le bal du Comte d'Orgel).

Type $2>\mathrm{N} 1<\mathrm{X}>\mathrm{V}$ à $\mathrm{N} 2<\mathrm{Y}>$ de $\mathrm{INF}<\mathrm{A}>$

(REQUETE)

(12) ... la mère permet à son enfant de sortir dans cette tenue d'enfant prostituée (M. DURAS, 1984, L'amant).

L'objectif est de voir quels verbes apparaissent au sein de ces cadres. Le langage de requêtes dans la partie catégorisée de la base FRANTEXT permet de rechercher des cadres prédicatifs nus (sans mention du verbe). Les résultats obtenus proviennent de cette base pour la période 1900-2000.

IV.1. Type $1 a: N 1_{<X>} V N 2_{<Y>} \grave{a} I N F_{<A>}$

Pour le type $1 \mathrm{a}$, on dénombre 216 occurrences mettant en jeu 28 verbes ${ }^{9}$. Les modalités ${ }^{10}$ rencontrées sont de deux types :

\footnotetext{
${ }^{9}$ Les verbes de production de parole sont suivis du symbole $\mathrm{P}$.
} 
[I] Interactions entre X et $\mathrm{Y}$ pour accomplissement de A $(94,9 \%)$

-3 verbes couvrent chacun plus de $10 \%$ du corpus (obliger, aider et inviter $^{\mathrm{P}}$ ); -4 verbes couvrent entre 5 et $10 \%$ du corpus (amener, contraindre, conduire et pousser) ;

-16 verbes couvrent moins de $5 \%$ du corpus inciter $^{\mathrm{P}}$, forcer, autoriser ${ }^{\mathrm{P}}$, encourager ${ }^{\mathrm{P}}$, décider ${ }_{\mathrm{P}}$, engager ${ }_{\mathrm{P}}^{\mathrm{P}}$, appeler ${ }_{\mathrm{P}}$, convier ${ }^{\mathrm{P}}$, condamner ${ }_{\mathrm{P}}^{\mathrm{P}}$, dresser, exciter, préparer, convoquer ${ }^{\mathrm{P}}$, déterminer, habituer, mener).

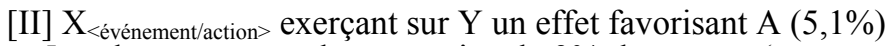

5 verbes couvrent chacun moins de $3 \%$ du corpus (porter, incliner, solliciter, exposer, induire). Représentant $5,1 \%$ du corpus total, ce deuxième cas reste cependant marginal face à l'écrasante présence des interactions pour accomplissement qui couvrent $94,9 \%$ du corpus.

On peut donc en conclure que, contrairement aux types qui suivent, le cadre prédicatif $\mathbf{N} 1_{<\mathbf{X}}>\mathbf{V} \mathbf{N} \mathbf{2}_{<\mathbf{Y}}: \mathbf{q q n}>\grave{\boldsymbol{a}} \mathbf{I N F} \mathbf{F}_{<\mathbf{A}}>$ est, sur la base de ce corpus, une véritable «CONSTRUCTION PREDICATIVE » car A constitue toujours une action à accomplir (et non déjà accomplie) et c'est Y qui est destiné à devenir l'agent de l'action A (en termes syntaxiques, c'est N2 qui contrôle l'infinitive).

IV.2. Type $1 b: N 1_{<X>} V N 2_{<Y>}$ de $I N F_{<A>}$

Pour le type $1 \mathrm{~b}$, on relève 1027 résultats concernant 41 verbes. Le verbe $\mathrm{em}$ pêcher se détache nettement des autres avec 438 occurrences. Viennent ensuite prier (139) et supplier (71). On note également que sur 41 verbes, 27 ont une fréquence d'apparition inférieure à $1 \%{ }^{11}$.

Les modalités rencontrées sont nettement plus nombreuses. On en relève 4 :

[I] Interactions pour accomplissement de A $(39 \%)^{12}$

Les interactions pour accomplissement de A sont de deux types :

i. C'est Y qui est appelé à accomplir A. Excepté prier $^{\mathrm{P}}(13,5 \%)$ et supplier $^{\mathrm{P}}$ $(6,9 \%)$, les autres verbes associés à cette modalité ont une fréquence d'apparition inférieure à $4 \%$ (presser, charger, forcer, obliger, persuader ${ }_{\mathrm{P}}^{\mathrm{P}}$, sommer $_{\mathrm{P}}{ }_{\mathrm{P}}$ contraindre, adjurer ${ }_{\mathrm{P}}$, solliciter ${ }_{\mathrm{P}}$, conjurer ${ }_{\mathrm{P}}$, défier ${ }_{\mathrm{P}}$, convaincre ${ }^{\mathrm{P}}$, implorer $^{\mathrm{P}}$, avertir ${ }^{\mathrm{P}}$, autoriser ${ }^{\mathrm{P}}$, convier ${ }^{\mathrm{P}}$, exhorter ${ }^{\mathrm{P}}$, requérir ${ }^{\mathrm{P}}$, tenter).

\footnotetext{
${ }_{11}^{10}$ Le type $[\mathrm{II}]$ constitue une extension de sens extralinguistique du type [I]

11 par fréquence décroissante : contraindre, envier, adjurer, solliciter, conjurer, défier, dissuader, menacer, féliciter, remercier, garder, convaincre, implorer, préserver, assurer, avertir, excuser, louer, récompenser, autoriser, convier, décourager, délivrer, exhorter, glorifier, requérir, tenter

ou marginalement effet d'une action ou d'une situation $\mathrm{X}$ sur $\mathrm{Y}$ favorisant l'accomplissement de A (cf. tenter)
} 
ii. C'est $\mathrm{X}$ qui est appelé à accomplir $\mathrm{A}$. Ce cas concerne uniquement le verbe menacer $^{\mathrm{P}}(0,6 \%)$.

[II] Interactions contre l'accomplissement de A $(49,1 \%)^{13}$

Les interactions contre l'accomplissement de A concernent $49,1 \%$ du corpus en raison de la présence écrasante d'un seul verbe, empêcher, qui couvre à lui seul $42,6 \%$ du corpus. Les 8 autres verbes qui relèvent de cette modalité ont une fréquence d'apparition inférieure à $3 \%$ (dispenser ${ }^{\mathrm{P}}$, retenir, détourner ${ }^{\mathrm{P}}$, dissuader $^{\mathrm{P}}$, garder, préserver, décourager ${ }^{\mathrm{P}}$, délivrer $)$.

[III] Jugements à propos de A accomplie ou en cours d'accomplissement $(11,7 \%)$. Les verbes accuser ${ }^{\mathrm{P}}(5,8 \%)$ et soupçonner ${ }^{\mathrm{P}}(3,3 \%)$ sont représentatifs de cette modalité, les autres verbes ayant une fréquence d'apparition très faible $(<1 \%)$ envier, excuser ${ }^{\mathrm{P}}$, féliciter ${ }^{\mathrm{P}}$, glorifier ${ }^{\mathrm{P}}$, ,ouer $^{\mathrm{P}}$, récompenser, remercier ${ }^{\mathrm{P}}$.

[IV] Déclarations à propos de $\mathrm{A}$ à accomplir $(0,2 \%)$ : Seul le verbe assurer $^{\mathrm{P}}$ $(0,2 \%)$, qui n'apparaît que deux fois, relève de cette modalité.

Le fait que les interactions contre l'accomplissement de A l'emportent (49,1\% vs. 39\%) pour ce cadre prédicatif est dû à notre méthode de classement basée sur les fréquences d'apparition de chaque verbe. Si l'on accordait le même poids à chaque verbe représenté, ce cadre prédicatif exprimerait en priorité une interaction en faveur de l'accomplissement de A (26 verbes sur 41). Il paraît donc délicat de qualifier ce cadre de "CONSTRUCTION PREDICATIVE » en raison du poids excessif d'un seul verbe. Pour justifier la prise en compte des fréquences, un argument psycholinguistique serait que le verbe empêcher fournirait le prototype sémantique de ce cadre. Mais selon Goldberg (2002 :348), les constructions permettent typiquement un éventail d'interprétations étroitement liées, or c'est bien le cas ici, car les deux interprétations ont en commun qu'une action SUSCEPTIBLE D'ETRE ACCOMPLIE est en cause et que le second partenaire Y est L'AGENT DESIGNE pour l'accomplissement ou le non accomplissement de cette action.

\section{IV.3. Type $2: N 1<X>V$ à $N 2<Y>$ de $I N F<A>$}

Les 159 occurrences rencontrées touchent 22 verbes dont les plus fréquents sont permettre (68 occurrences) et demander ${ }^{\mathrm{P}}(22)$. Les autres verbes concernent chacun moins de $6 \%$ du corpus (ordonner ${ }^{\mathrm{P}}$, dire ${ }_{\mathrm{P}}^{\mathrm{P}}$, proposer ${ }_{\mathrm{P}}$, reprocher $\mathrm{P}_{\mathrm{P}}$, conseiller $^{\mathrm{P}}$, interdire ${ }^{\mathrm{P}}$, imposer, prescrire ${ }^{\mathrm{P}}$, défendre ${ }^{\mathrm{P}}$, offrir ${ }^{\mathrm{P}}$, recommander ${ }^{\mathrm{P}}$, suggérer $^{\mathrm{P}}{ }_{\mathrm{P}}$ commander $^{\mathrm{P}}$, devoir, dicter ${ }^{\mathrm{P}}$, donner, empêcher, enjoindre ${ }^{\mathrm{P}}$, éviter, promettre $^{\mathrm{p}}$.

Cette fois, les modalités rencontrées se répartissent en trois groupes : 13 ou marginalement effet d'une action ou d'une situation X sur Y favorisant le non ac-
complissement de A (cf. délivrer) 
[I] Interaction pour l'accomplissement de A $(88,1 \%)$ qui, comme pour le cadre $1 \mathrm{~b}$, se subdivise en deux sous-classes :

i. Accomplissement de A par Y $(87,5 \%)$. Le verbe permettre couvre à lui seul $42,8 \%$ du corpus. Excepté demander qui couvre 13,8\% du corpus, les autres verbes ont une fréquence d'apparition de moins de $6 \%$ (ordonner ${ }^{\mathrm{P}}$, di$r^{\mathrm{P}}$, proposer ${ }^{\mathrm{P}}$, conseiller ${ }_{\mathrm{P}}$, imposer, prescrire, offrir $^{\mathrm{P}}$, recommander ${ }_{\mathrm{P}}$, suggérer ${ }_{\mathrm{P}}$, commander ${ }^{\mathrm{P}}$, dicter ${ }^{\mathrm{P}}$, donner, enjoindre ${ }^{\mathrm{P}}$ ).

ii. Accomplissement de A par X qui concerne un seul verbe promettre $(0,6 \%)$.

[III] Interactions contre l'accomplissement de A (6,3\%). Cette modalité concerne 4 verbes (interdire ${ }^{\mathrm{P}}: 3,8 \%$, défendre ${ }^{\mathrm{P}}: 1,3 \%$, empêcher : $0,6 \%$, éviter : $0,6 \%$ ).

[IV] Autres (5,6\%). Deux verbes évoquent des modalités difficilement identifiables. Reprocher $(5 \%)$, tout d'abord ne peut pas vraiment être interprété comme relevant des interactions car il n'implique pas la présence de deux interlocuteurs et devoir $(0,6 \%)$, qui est encore plus marginal et délicat à classer.

Le type 2 présente une analogie avec le type $1 \mathrm{~b}:$ le verbe permettre assure à lui seul $42,8 \%$ des occurrences. Mais contrairement au type $1 \mathrm{~b}$, permettre ne fait que confirmer une tendance générale : la valeur dominante est une interaction EN FAVEUR de l'accomplissement de A par Y, que les fréquences soient prises en compte ou pas. On peut donc accorder à ce cadre le statut de « construction prédicative » (identique au type 1a) bien que 11,9\% des occurrences rencontrées aient un sémantisme différent (contre 5, 1\% pour le type 1a).

\section{CONCLUSION}

Le bilan de cette étude est clair: si un verbe primairement incompatible avec une infinitive se laisse greffer un cadre prédicatif comportant une infinitive, cette greffe a toute chance d'induire chez un locuteur natif la représentation d'une INTERACTION ENTRE DEUX PARTENAIRES X ET Y RESPECTIVEMENT EXPRIMES PAR LE SUJET ET L'OBJET (DIRECT OU PREPOSITIONNEL) VISANT L'ACCOMPLISSEMENT (OU LE NON ACCOMPLISSEMENT) DE $<$ A $>$ PAR $<$ Y $>$ OU L'ACCOMPLISSEMENT DE $<\mathrm{A}>$ PAR $<\mathrm{X}>$.

Dans le cadre théorique d'A. Goldberg, ces trois types de cadres prédicatifs présentent un même prototype sémantique sous trois variantes :

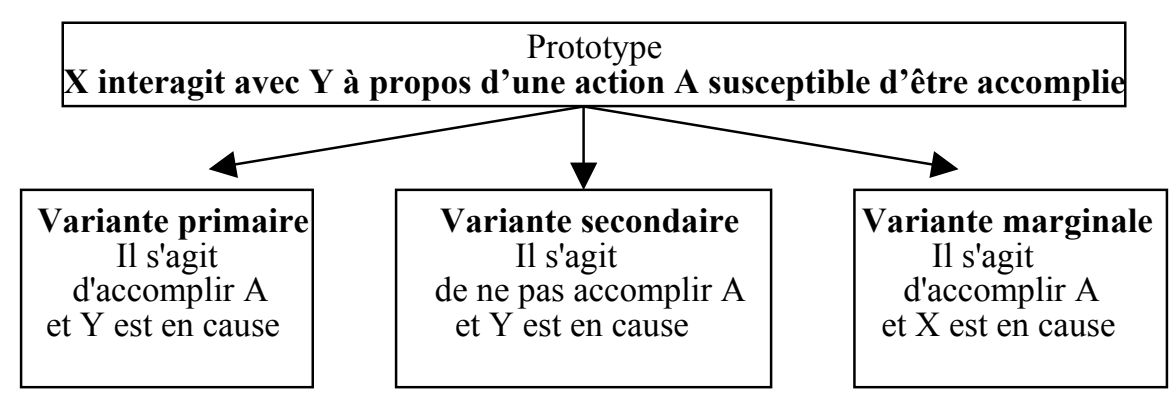


Les autres types d'emploi (déclaration, jugement et relation action-moyen) sont des dérivations périphériques de ce prototype.

\section{REFERENCES}

Croft W., 1998, The structure of events and the structure of language, in M. Tomasello (ed), The new psychology of language - Cognitive and functional approaches to language structure. Mahwah, NJ : LEA. p. 67-92.

Croft W., 2001, Radical Construction Grammar: Syntactic theory in typological perspective. Oxford : Oxford University Press.

Croft W. \& Cruse W.A., 2004, Cognitive Linguistics. Cambridge UK : Cambridge University Press.

Fillmore Ch., Kay P. \& Kay O’Connor M. 1988, Regularity and idiomaticity in grammatical constructions : the case of let alone, Language 64, p. 501-38.

François J., 2003, La prédication verbale et les cadres prédicatifs. Louvain : Peeters [Bibliothèque de l'Information Grammaticale 54]

Goldberg A., 1995, Constructions - A Construction Gramamr approach to argument structure. Chicago : Chicago University Press.

Goldberg A., 1998 Patterns of experience in patterns of language, in : M. Tomasello (ed), The new psychology of language - Cognitive and functional approaches to language structure. Mahwah, NJ : LEA, p. 203-19.

Goldberg A., 1999, The emergence of the semantics of argument structure constructions In : W. McWhinney ed., The emergence of language. Mahwah, New-Jersey, Chapter 7, p. 197-212.

Goldberg A., 2002, Surface generalizations: an alternative for alternations, Cognitive Linguistics 13-4, 327-356.

Kay P., 1997, Construction grammar feature structures revised. Consultable sur le site http://www.icsi.berkeley.edu/ kay/bcg/Fsrev.html.

Lakoff G., 1987, Women, fire and dangerous things. What categories reveal about the mind. Chicago : Chicago University Press.

Langacker R. 1987, 1991, Foundations of Cognitive Grammar. Vol.1 : Theoretical prerequisites. Vol.2: Descriptive applications. Standford: Standford University Press.

Sénéchal M., 2003, Les verbes et les prépositions : variations de sens et variations de constructions. Mémoire de DEA, Université de Caen.

Vanderveken D. 1988, Les actes de discours. Bruxelles : Mardaga.

Willems D. 2002, Classes et relations verbales. A la recherche d'une typologie intégrée. In : J. Chuquet \& M. Paillard (dir.), Morphosyntaxe du lexique-I : Catégorisation et mise en discours. Travaux linguistique du CerLiCO 15. Presses Universitaires de Rennes, p.285-302.

Willems D. 2005, Typologie des procès et régularités polysémiques, in D. Bouchard \& I. Evrard (dir.), Représentations du sens II, Travaux de Linguistique, 2005. 